Original Research Paper

\title{
Influence of The Extract of Brown Seaweed (Turbinaria decurrens Bory) on the Histology of Colon on AOM-DSS Mouse Model
}

\author{
Anton Bahtiar and Dian Anggraeni \\ Department of Pharmacology and Toxicology, Faculty of Pharmacy, Universitas Indonesia, Depok, Indonesia
}

\author{
Article history \\ Received: 29-11-2017 \\ Revised: 30-11-2017 \\ Accepted: 13-12-2017 \\ Corresponding Author: \\ Anton Bahtiar \\ Department of Pharmacology \\ and Toxicology, Faculty of \\ Pharmacy, Universitas \\ Indonesia, Depok, Indonesia \\ Tel/Fax : +62-21-7864049/+62- \\ $21-7863433$ \\ Email: anton.bahtiar@farmasi.ui.ac.id
}

\begin{abstract}
Utilization of seaweed as a commodity trading or as a raw materials industry is still relatively small compared with the diversity of seaweed species in Indonesia. Turbinaria decurrens Bory is one of the brown seaweed species that has a high enough fucoxanthin content compared with other brown seaweed species. Fucoxanthin is one of the carotenoid pigments that have long been known to have bioactivity as antiinflammatory, antioxidant and anticarcinogenic that can neutralize free radicals. Therefore, in this study, we evaluated another function of Turbinaria decurrens Bory as a candidate for colon cancer drug. To this end, we used 36 Male Swiss Webster mice aged 5-6 weeks and divided into six groups. Group I as normal groups, Group II as AOM-DSS induced cancer group, Group III as Aspirin treated group; Group IV-VI Brown Seaweed extracts dose 1 to 3 are $3.61 ; 7.22 ; 14.41 \mathrm{mg} / \mathrm{kg}$ body weight, respectively. All groups except normal group treated by $12.5 \mathrm{mg} / \mathrm{kg}$ body weight of AOM by intraperitoneal injection followed by two cycles of DSS treatment and five days for each treatment. At the end of the experiment, mice sacrificed and the colon was isolated and subject to histology treatment. As a result, showed that AOM-DSS could make colon cancer in all mice. The administration of brown seaweed extract at the dose I could prevent the development of cancer. Increasing dose could not prevent better, but it makes worst.
\end{abstract}

Keyword: Colon Cancer, AOM-DSS, Turbinaria decurrens Bory

\section{Introduction}

Indonesia is known as an archipelago country which has two-thirds of its territory is ocean and has the longest coastline in the world, it almost 81 thousand $\mathrm{km}$. One of the living things that grow and develop in the sea is seaweed. Utilization of seaweed as a commodity trading or raw materials industry is still relatively small when compared with the diversity of seaweed species in Indonesia. Some studies have shown that brown seaweed contains active compounds with a variety of bioactivity that has the potential to be developed with health goals, such as chlorophyll content, beta-carotene, violaxanthin and fucoxanthin (Mikami and Hosokawa, 2013). Research has shown that brown seaweed has potential as anti-inflammatory because it contains polysaccharide sulfate, PUFA and fucoxanthin (Jaswir and Monsur, 2011). Carotenoids from seaweed are also proven as powerful antioxidants and can prevent some degenerative diseases, cardiovascular and cancer. In the field of food also carotenoids have been applied as food colorants and supplements.

Turbinaria decurrens Bory is one of the brown seaweed that has a high enough of fucoxanthin content compared to other brown seaweed (Wen et al., 2016). Fucoxanthin is one of the carotenoid pigments that have a long time been known to have bioactivity as antiinflammatory, antioxidant and anticarcinogenic that can neutralize free radicals. Fucoxanthin content on Turbinaria decurrens Bory is $86.9 \mathrm{mg} / \mathrm{g}$ extract and has a cytotoxic potential of 56\% (Wu et al., 2016).

Colon cancer is cancer that occurs inside or on the surface of the colon. In 2008 worldwide there were more than 1 million colon cancer incidents with mortality rates over 50\% (Jaswir et al., 2013). The high incidence of colon cancer in Indonesia is closely related to one risk factor, namely lifestyle such as diet, weight and physical activity. 
The Azoxymethane-Dextran Sodium Sulfate (AOMDSS) induced mouse model is a typical inflammationrelated cancer model in which adenoma and adenocarcinoma are induced using an intraperitoneal injection of a low dose of AOM followed by oral administration of DSS. This model is therefore widely accepted for the study of colon carcinogenesis, especially for inflammation-related carcinogenesis.

From this background, a study was conducted to examine the anti-inflammatory effects of brown seaweed extract (Turbinaria decurrens Bory.) in male colon cancer mice.

\section{Materials and Methods}

Plant and Chemical Materials

Brown Seaweed (Turbinaria decurrens Bory) collected from Binuangen Beach, Banten, Indonesia. The collected seaweeds were washed with tap water and distilled water to remove salt and other debris then packed with ice to keep cool during the transportation to the laboratory. Extraction of Brown Seaweed was carried out in the laboratory of Indonesian Center for Spices and Medicinal Plants Research, Bogor. The extractive value of ethanolic extract of Brown seaweed calculated as \% w/w yield and was found to be $1.4 \%$. The identification of the extracts conducted in the laboratory of phytochemistry, Faculty of Pharmacy, Universitas Indonesia

\section{Animals}

Male Swiss Webster mice aged 5-6 weeks, were purchased from National Institute of Health Research and Development - NIHRD. The rats were grouped and housed in cages with one animal per cage and maintained under temperature $25 \pm 2{ }^{\circ} \mathrm{C}$ with dark and light cycle $(12 / 12 \mathrm{~h})$ and allowed free access to a commercial pellet diet and water ad libitum.

Mice were acclimatized to laboratory condition for one week before the commencement of the experiment. This research had certified by ethical certification of Faculty of Medicine, University of Indonesia (UI FM No. 214/UN2.F1/Ethics/2017) for the use of animals in experiments.

Establishment of the Azoxymethane - Dextran Sodium Sulphate (AOM-DSS) Colon Cancer model

AOM-DSS model mice were established based on the methods that published in the previous report, with some modification. Briefly, six male Swiss Webster mice (seven weeks old) were intraperitoneally injected with AOM (Sigma-Aldrich, St. Louis, USA) at $12.5 \mathrm{mg} / \mathrm{kg}$ body weight. One week after AOM injection, the mice divided into six group. All six group were treated with $2.5 \%$ of DSS (Sigma-Aldrich, St. Louis, USA) for five days then resting state for 15 days then follow with the second cycle of DSS for five days followed by 15 days of normal water.

\section{Identification of Fucoxanthin by Thin Layer Chromatography}

The identification of fucoxanthin by TLC using a mobile phase of n-hexane: Acetone (6: 4) (Su et al., 2016). The plate heated in the oven for $2 \mathrm{~h}$ then the crude extracts and the standard of fucoxanthin were each dissolved into ethanol and sterilized into plates with a spacing of $0.5 \mathrm{~cm}$ each. The plates inserted into a chamber that has already saturated for $3 \mathrm{~h}$ with an $\mathrm{n}$ hexane solution: Acetone (6:4). The plate removed from the chamber when the solvent movement reaches the upper limit of the plate. The content of the fucoxanthin identified by determining the value of the Retardation Factor (Rf) derived from the distance of the sample movement from the starting point and the distance of the solvent from the starting point.

\section{Extract of Turbinaria Decurrens Bory Treatment}

The extract was administered to the mice at the beginning of the research, so we started before we induced by AOM for 49 days until 2nd cycles of DSS ended. We administered the mice in three various doses namely dose $1=3.61 \mathrm{mg} / \mathrm{kg}$ body weight, dose $2=7.22$ $\mathrm{mg} / \mathrm{kg}$ body weight and dose $3=14.41 \mathrm{mg} / \mathrm{kg}$ body weight. As a positive control, we use celecoxib $26 \mathrm{mg} / \mathrm{kg} \mathrm{BW}$.

\section{Macroscopic and Histopathological Evaluation}

At the end of the experiment, mice sacrificed by cervical dislocation to evaluate macroscopically of the colon. Then, colon tissue from each mouse was dissected into $0.3 \mathrm{~cm}^{2}$ pieces and carefully rinsed with PBS containing with PBS 3 times. The middle and distal colons were dissected out, opened longitudinally and stain by alcian blue to determine the number and size of tumors.

To evaluate histology of colon, after stained by alcian blue for macroscopic evaluation, then rinse by PBS and fixed using neutral buffered formalin overnight before H\&E staining.

\section{Results}

\section{Identification of Fucoxanthin by Thin Layer Chromatography}

We evaluated the content of the fucoxanthin on the brown seaweed extract by thin layer chromatography. And the result as shown in Fig. 1.

As shown in Fig. 1, fucoxanthin identified as yellow to orange color in visible light. In the visible light and UV366 $\mathrm{nm}$, we found that similar spot in the extract with a single spot in the fucoxanthin standard with RF 0.78. 


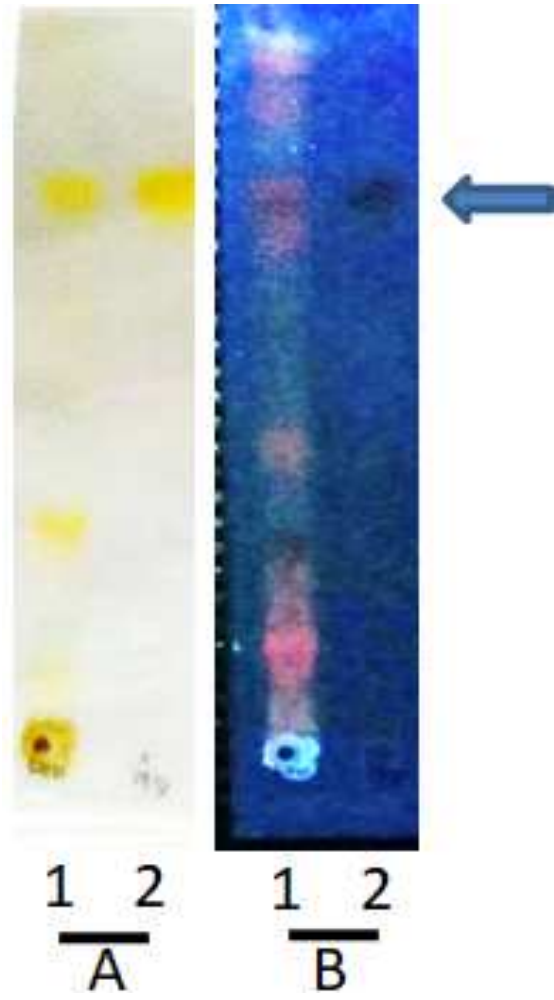

Fig. 1: Thin layer chromatography of brown seaweed extracts. Note: (A) Identification by visible light (B) Identification on UV360nm; 1. Brown seaweed extracts; 2. Standard fucoxanthin; Blue arrow indicated Fucoxanthin

\section{Macroscopic View of Colon AOM-DSS Treated Mice}

The inflammation-related carcinogenesis model mouse induced by AOM-DSS was implemented by initially injecting the mice with AOM and then periodically feeding the mice by DSS. During each DSS cycle, the mice displayed diarrhea and blood in feces at the end of DSS feeding period; all these symptoms were attenuated gradually since the mice fed with normal water. The results of fecal occult blood tests were negative at the end of each DSS cycle (Su et al., 2016). The mice who went through different treatments sacrificed and changes in their colonic morphology were examined and compared. The result as shown in Fig. 2.

Figure 2 showed that AOM-DSS treatment caused many tumors at the distal of the colon (2C) compared with normal mice (2A). The administration of celecoxib as positive control seemed to prevent the development of the tumor. The administration of brown seaweed apparently inhibit tumor growth but they did not show dose dependency and dose 1 seems to inhibit better than a higher dose.

\section{Histology of Colon AOM-DSS Treated Mice}

In Fig. 3, AOM-DSS treatment caused dysplasia cell (3B) compared with normal group (3A). The administration of Celecoxib prevents the changes size and shape of the cell. The administration of Brown seaweed extract similar to Celecoxib.
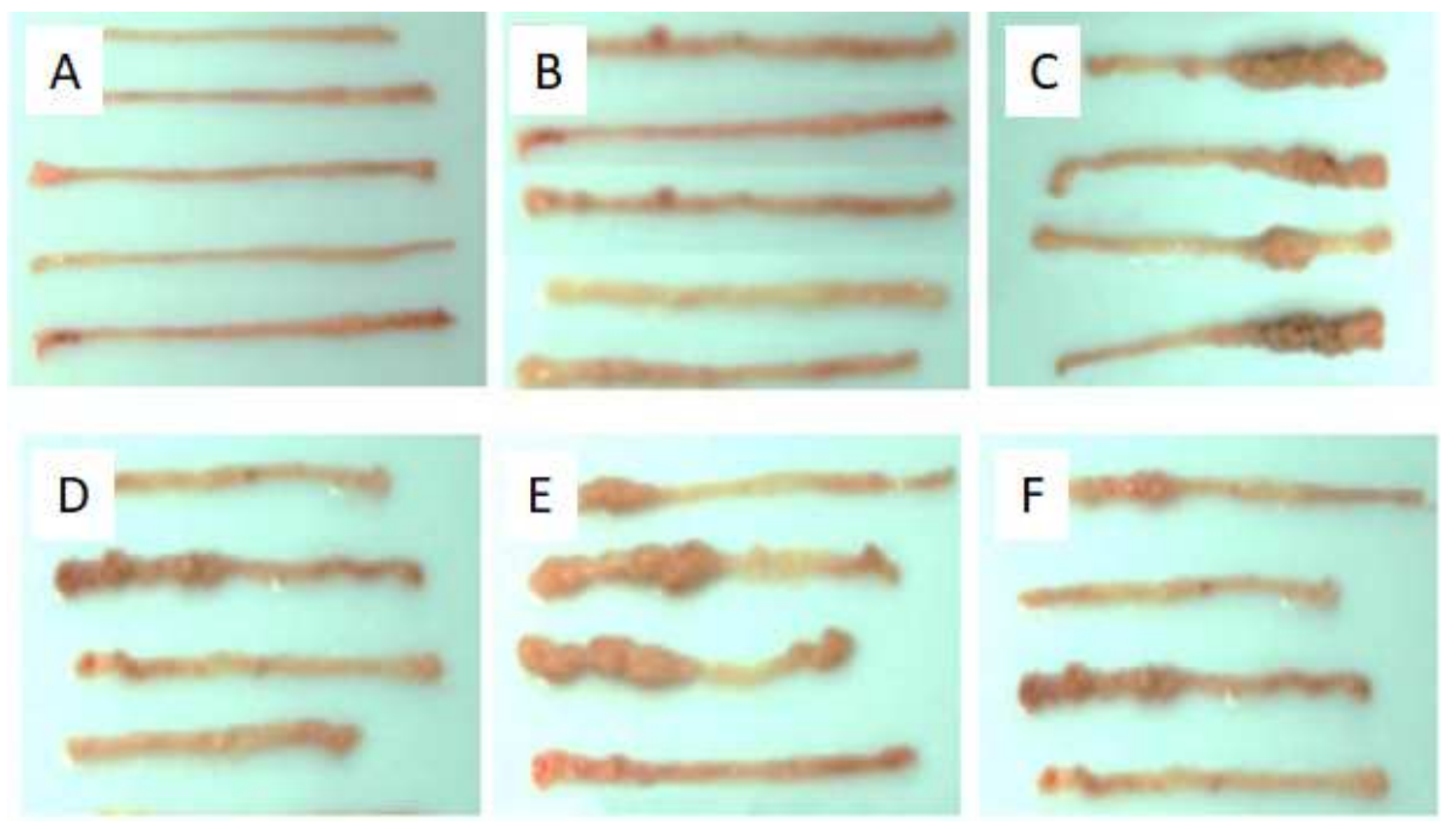

Fig. 2: The macroscopic of the colon after isolated and stain by Alcian Blue. (A) Normal group (B) Positive control group (C) Negative control group (D) Dose 1 of TDE (E) Dose 2 of TDE (F) Dose 3 of TDE 

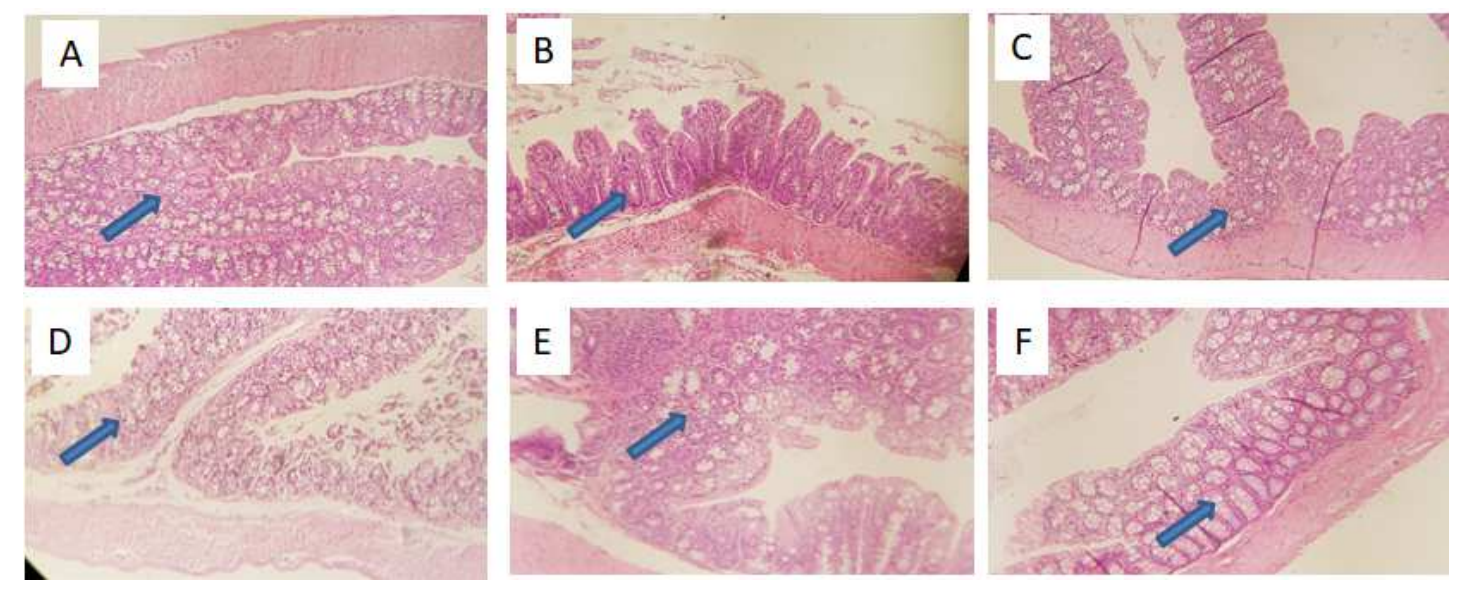

Fig. 3: The histology of colon during treatment. A. Normal group; B. Negative control group; C. Positive control group; D. Dose 1 of TDE; E. Dose 2 of TDE; F. Dose 3 of TDE; TDE = Turbinaria decurrens extract; blue arrow: Dysplasia cell

\section{Discussion}

We identified fucoxanthin in our Brown seaweed extracts by TLC. Identification of compounds using TLC based on color spot and $\mathrm{Rf}$ value. The movement of the alleged fucoxanthin compound is present in the spot a sample of extract and a standard fucoxanthin with an orange spot and a distance value of the compound movement has similar Rf 0.78 . In previous reported, identification of brown seaweed by TLC showed carotenoid groups, especially fucoxanthin identified as an orange color (Mikami and Hosokawa, 2013). The value of Rf fucoxanthin on brown seaweed using mobile phase of n-hexane: Acetone (6: 4) was 0.78 .

Although there are a variety of chemical agents available to induce colon carcinogenesis in animals, AOM is widely used due to its practical advantages, such as reproducibility, high potency, simple application, excellent stability and a low price (Wang et al., 2015). AOM converted to methylazoxymethanol by CYP450 2E1 in the liver. The AOM metabolite, Methylazoxymethanol, could induce toxicity to the gen various tissues including the colon. The DSS, a pro-inflammatory stimulus, is used to induce colitis in animals. Continuous exposure to DSS induces inflammatory conditions in the colon. When AOM combined with the pro-inflammatory stimulus DSS, the tumor growth was accelerated with a significantly shorter latency time (Rosenberg et al., 2009). AOM-DSS induced inflammation through cytokines that induced inflammation and finally becomes colon cancer. The cytokines that involve in the colon carcinogenesis are TNF- $\alpha$, IL-1 $\beta$, IL-6, COX-2, iNOS, estrogen receptor- $\beta, \quad \beta$-catenin, 5lipoxygenase and others (Janakiram and Rao, 2008).

In the previous study, fucoxanthin showed suppresses the inflammation of Endotoxin-Induced Uveitis (EIU) rats by blocking the iNOS and COX-2 protein expression (Ilieva et al., 2004). Therefore, fucoxanthin could prevent cancer development because could inhibit the cytokine that produced by AOM-DSS.

\section{Conclusion}

According to above data, we concluded that Brown seaweed could develop as a promising drug for colon cancer.

\section{Acknowledgement}

This work was supported by Directorate Research and Community Service, Universitas Indonesia 2016.

\section{Author's Contributions}

Anton Bahtiar: Designed the research plan and organized the study, contributed to the writing of the manuscript.

Dian Anggraeni: Coordinated the mouse work, participated in all experiments, coordinated the dataanalysis.

\section{Ethics}

This research had certified by ethical certification of Faculty of Medicine, University of Indonesia (UI FM No. 214/UN2.F1/Ethics/2017) for the use of animals in experiments.

\section{References}

Mikami, K. and M. Hosokawa, 2013. Biosynthetic pathway and health benefits of fucoxanthin, an algae-specific xanthophyll in brown seaweeds. Int. J. Mol. Sci., 14: 13763-13781.

Jaswir, I. and H.A. Monsur, 2011. Review antiinflammatory compounds of macro seaweed origin: A review. J. Medicinal Plants Res., 5: 7146-54 
Wen, Z.S., X.W. Xiang, H.X. Jin, X.Y. Guo and L.J. Liu et al., 2016. Composition and antiinflammatory effect of polysaccharides from Sargassum horneri in RAW264.7 macrophages. Int. J. Biol. Macromol., 88: 403-413.

DOI: $10.1016 /$ j.ijbiomac.2016.02.025

Wu, G.J., S.M. Shiu, M.C. Hsieh and G.J. Tsai, 2016. Antiinflammatory activity of a sulfated polysaccharide from the brown alga Sargassum cristaefolium. Taiwan: Food Hydrocolloids, 53: 16-23.

Jaswir, I., D. Noviendri and H.M. Salleh, 2013. Analysis of fucoxanthin content and purification of all-transfucoxanthin from Turbinaria turbinate and Sargassum plagyophyllum by $\mathrm{SiO} 2$ open column chromatography and reversed phase-HPLC. J. Liquid Chromatography Related Technol., 36: 1340-1354.

Su, C., Y. Zhang and Y. Zhu, 2016. Stromal COX-2 signaling is correlated with colorectal cancer: A review. Critical Rev. Oncology/Hematol., 107: 33-38. DOI: 10.1016/j.critrevonc.2016.08.010
Wang, Y., Q. Shan, G. Hou, J. Zhang and J. Bai et al., 2015. Discovery of potential colorectal cancer serum biomarkers through quantitative proteomics on the colonic tissue interstitial fluids from the AOM - DSS mouse model. J. Proteomics, 132: 31-40. DOI: $10.1016 /$ j.jprot.2015.11.013

Rosenberg, D.W., C. Giardina and T. Tanaka, 2009. Mouse models for the study of colon carcinogenesis. Carcinogenesis, 30: 183-196. DOI: $10.1093 /$ carcin/bgn267

Janakiram, N.B. and C.V. Rao, 2008. Molecular markers and targets for colorectal cancer prevention. Acta Pharmacol Sin, 29: 1-20. DOI: $10.1111 /$ j.1745-7254.2008.00742.x

Ilieva, I., K. Ohgami, K. Shiratori, Y. Koyama and K. Yoshida et al., 2004. The effects of Ginkgo biloba extract on lipopolysaccharide-induced inflammation in vitro and in vivo. Exp. Eye Res., 79: 181-187. DOI: 10.1016/j.exer.2004.03.009 